\title{
Efecto de Acacia macracantha en las propiedades físicas y químicas del suelo en un sistema silvopastoril
}

\author{
Effect of Acacia macracantha on the physical and chemical properties \\ of soil in a silvopastoral system
Gelver Romero Delgado ${ }^{1}$, Fritz Trillo Zárate ${ }^{2}$, Javier Orellana Chirinos ${ }^{1}$, Pamela Quiroga Espilco ${ }^{3}$, Jorge Gamarra Bojorquez ${ }^{4}$, David Rojas Egoavil ${ }^{5}$, Virginia Rivadeneira ${ }^{6}$, Jimny Nuñez Delgado ${ }^{6,7}$

\section{Resumen}

\begin{abstract}
El objetivo del presente estudio fue determinar el efecto de dos edades del árbol faique (Acacia macracantha) sobre las características físicas y químicas del suelo en un sistema silvopastoril con pasto castilla (Panicum maximum Jacq). El estudio se realizó en el distrito de Bellavista, Cajamarca, Perú. Se evaluó la resistencia mecánica, densidad aparente, humedad y temperatura del suelo, así como el contenido de materia orgánica, fósforo $\left(\mathrm{P}_{2} \mathrm{O}_{5}\right)$ y potasio $\left(\mathrm{K}_{2} \mathrm{O}\right)$ en tres ubicaciones del suelo: bajo la copa de árboles jóvenes (BCAj) y medianos (BCAm) y fuera de la copa del árbol (FCA), en dos épocas del
\end{abstract}

${ }^{1}$ Laboratorio de Ecología y Utilización de Pastizales, Facultad de Zootecnia, Universidad Nacional Agraria la Molina, Lima, Perú

${ }^{2}$ Departamento de Producción Animal, Facultad de Zootecnia, Universidad Nacional Agraria la Molina, Lima, Perú

${ }^{3}$ Laboratorio de Análisis de Suelos, Plantas, Agua y Fertilizantes, Facultad de Agronomía, Universidad Nacional Agraria la Molina, Lima, Perú

${ }^{4}$ Departamento de Nutrición Animal, Facultad de Zootecnia, Universidad Nacional Agraria la Molina, Lima, Perú

${ }^{5}$ Unidad de Investigación Agropecuaria, IVITA-Mantaro, Facultad de Medicina Veterinaria, Universidad Nacional Mayor de San Marcos, Huancayo, Perú

${ }^{6}$ Departamento de Producción Animal, Facultad de Medicina Veterinaria, Universidad Nacional Mayor de San Marcos, Lima, Perú

${ }^{7}$ E-mail: jnunezd@unmsm.edu.pe; https://orcid.org/0000-0001-6310-418X

Recibido: 5 de marzo de 2020

Aceptado para publicación: 18 de noviembre de 2020

Publicado: 23 de junio de 2021

CLos autores. Este artículo es publicado por la Rev Inv Vet Perú de la Facultad de Medicina Veterinaria, Universidad Nacional Mayor de San Marcos. Este es un artículo de acceso abierto, distribuido bajo los términos de la licencia Creative Commons Atribución 4.0 Internacional (CC BY 4.0) [https:// creativecommons.org/licenses/by/4.0/deed.es] que permite el uso, distribución y reproducción en cualquier medio, siempre que la obra original sea debidamente citada de su fuente original 
año (seca y lluvia). Se utilizó un diseño completo al azar con arreglo factorial 3 (ubicación) x 2 (época). Bajo la copa de los árboles medianos se encontró una menor temperatura del suelo $\left(27.96^{\circ} \mathrm{C}\right)$ y mayor humedad del suelo $(15.23 \%)$, además de mejorar la fertilidad del suelo. El estudio permitió determinar que la presencia de los árboles en estos sistemas silvopastoriles mejora las condiciones físicas y químicas del suelo, además generan un microclima, mejorando las condiciones ambientales.

Palabras clave: sistema silvopastoril, humedad del suelo, copa de árbol, pastura

\section{AbSTRaCT}

The aim of this study was to determine the effect of two ages of the long-spine acacia tree (Acacia macracantha) on the physical and chemical characteristics of the soil in a silvopastoral system with Guinea grass (Panicum maximum Jacq). The study was conducted in the Bellavista district, Cajamarca, Peru. The mechanical resistance, apparent density, humidity and temperature of the soil, as well as the content of organic matter, phosphorus $\left(\mathrm{P}_{2} \mathrm{O}_{5}\right)$ and potassium $\left(\mathrm{K}_{2} \mathrm{O}\right)$ were evaluated in three soil locations: under the canopy of young (BCAj) and medium trees (BCAm) and outside the tree canopy (FCA), in two seasons of the year (dry and rainy). A complete randomized design with factorial arrangement 3 (location) $\times 2$ (seasons) was used. Under the canopy of the medium trees, a lower soil temperature $\left(27.96^{\circ} \mathrm{C}\right)$ and higher soil humidity $(15.23 \%)$ were found, in addition to improving soil fertility. The study made it possible to determine that the presence of trees in these silvopastoral systems improves the physical and chemical conditions of the soil, and generates a microclimate, improving environmental conditions.

Key words: silvopastoral system, soil humidity, canopy, pasture

\section{INTRODUCCIÓN}

En las regiones tropicales es frecuente la tala y quema de los bosques para el establecimiento de cultivos y posteriormente pasturas, lo cual trae consigo que los suelos desnudos pierdan su calidad, productividad y diversidad, dado que quedan susceptibles a procesos erosivos (Oliva, 2016). Los sistemas silvopastoriles (SSP) son una alternativa en la producción ganadera tropical a nivel mundial, debido a que permiten el uso sostenible de los bosques, pues no se pierde la capacidad productiva del suelo (Lemaire et al., 2014; Moraes et al., 2014).
Los árboles presentan un gran potencial para la recuperación de suelos con problemas de fertilidad (Alegre et al., 2012), debido al aumento de la entrada de materia orgánica. Las leguminosas arbóreas reducen las pérdidas de materia orgánica y nutrientes a través del ciclaje y control de la erosión, mejorando las propiedades físicas del suelo, la retención de agua y presentando un efecto benéfico sobre los procesos microbiológicos (FAO, 1992). Además, estos sistemas parecen que presentaran mayor resiliencia para compensar las posibles pérdidas de producción, debido a la ocurrencia episódica de cambios meteorológicos extremos atribuibles al cambio climático (Dagang y Nair, 2003). 
La incorporación del componente arbóreo en un sistema agrícola contribuye a la productividad del sistema proporcionando beneficios tales como: mejora de las condiciones del suelo (por ejemplo, secuestro de carbono orgánico), madera, fruta, forraje y un hábitat para la fauna silvestre (Andrade et al., 2008). Por otro lado, mejora el bienestar animal y permite un mayor rendimiento económico (Yates et al., 2007).

El objetivo del presente estudio fue evaluar el estado actual de las propiedades físicas y químicas del suelo en un sistema silvopastoril donde el componente arbóreo es la Acacia macracantha y el componente pasto es el monocultivo Panicum maximum Jacq.

\section{Materiales y MéTodos}

El presente estudio se realizó en un fundo representativo del distrito de Bellavista, provincia de Jaén, departamento de Cajamarca, Perú, clasificado como selva alta, ya que se encuentra a una altitud de $729 \mathrm{msnm}$ (5392 473 latitud S $78^{\circ} 402453$ longitud O) (Figura 1).

El lugar de estudio tuvo un relieve plano. Se evaluaron 54 ha divididas en 14 potreros con cercos fijos. Se eligieron aleatoriamente siete potreros distribuidos espacialmente de manera de cubrir el total del área evaluada. El componente pasto fue el monocultivo de pasto castilla (Panicum maximum Jacq), naturalizado (establecido hace 20 años). El componente arbóreo fue la especie nativa Faique (Acacia macracantha), de característica caducifolia, con hojas bipinnadas y poco densas, donde los rayos del sol atraviesan con facilidad. La densidad promedio fue 81 árboles por hectárea. El componente animal estuvo representado por ganado vacuno criollo doble propósito bajo pastoreo rotacional (dos meses de descanso) durante el estudio.
Para la categorización de los árboles se utilizó el método diámetro a la altura del pecho (DAP; FAO, 2004). El método consiste en medir la circunferencia $(\mathrm{C})$ a la altura del pecho $(1.3 \mathrm{~m})$ mediante una cinta métrica, donde el diámetro se determinó con la fórmula $\mathrm{D}=\mathrm{C} /$, donde $\mathrm{C}$ : circunferencia $(\mathrm{cm})$ у $: 3.1416$.

\section{Tratamientos}

Los árboles fueron clasificados según el DAP, obteniéndose la siguiente distribución: árboles en regeneración $(<7.5 \mathrm{~cm}$ DAP) $13.58 \%$, jóvenes (7.5-24 DAP) $50.62 \%$, pequeños (25-37 DAP) $17.28 \%$ y medianos (3849 DAP) $18.52 \%$.

Se utilizaron siete potreros de aproximadamente 2 ha cada uno. Los tratamientos fueron con base a las dos categorías de árbol con mayor densidad: fuera de la copa de los árboles (FCA), bajo la copa del árbol joven (BCAj) y bajo la copa del árbol mediano (BCAm). Cada tratamiento tuvo tres repeticiones (calicatas de $1 \times 0.5 \times 0.5 \mathrm{~m}$ ). El muestreo se realizó en las épocas de lluvia (marzo) y seca (agosto). La temperatura ambiental se midió a $1.5 \mathrm{~m}$ sobre la superficie del suelo en cada tratamiento, utilizando un termómetro ambiental digital (Deltatrak 12207, precisión: $\left.\pm 1.0^{\circ} \mathrm{C}\right)$.

\section{Evaluación del Suelo}

\section{Densidad aparente}

La densidad aparente para cada calicata se determinó mediante un cilindro muestreador de acero (5.29 de alto y 4.89 cm de diámetro) (Grossman y Reinsch, 2002). Se tomaron tres muestras del perfil del suelo cada $10 \mathrm{~cm}$. La densidad aparente fue determinada mediante la fórmula $\mathrm{Da}=\mathrm{Ms} / \mathrm{Vt}$, donde Da: densidad aparente $\left(\mathrm{g} / \mathrm{cm}^{3}\right)$; Ms: masa de suelo seco (g); Vt: volumen total del cilindro $\left(\mathrm{cm}^{3}\right)$. 

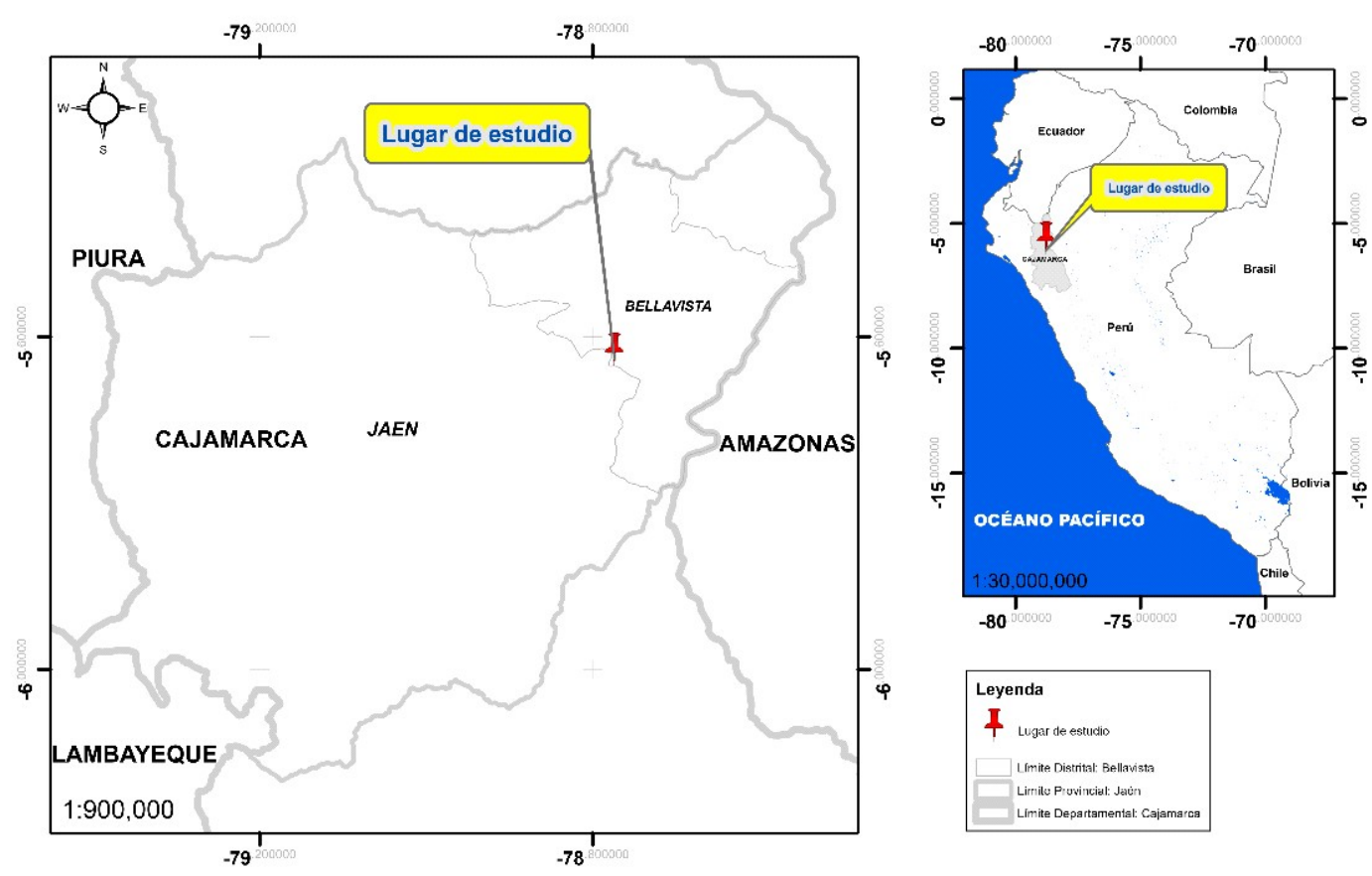

Figura 1. Ubicación geográfica del lugar de estudio

La porosidad se estimó mediante los datos de retención de agua y densidad aparente. La porosidad total del suelo fue calculada en base a los datos de la densidad aparente y real, según $\% \mathrm{Pt}=[1-(\mathrm{Dap} / \mathrm{Dr})]$ x 100 , donde \%Pt: Porcentaje de porosidad total; Dap: Densidad aparente: Dr: Densidad real.

\section{Resistencia mecánica}

Para medir la resistencia mecánica se utilizó un penetrómetro de bolsillo con pistón de carga de acero inoxidable (ELE International ${ }^{\circledR}$, EI29-3729), con escala de $0.25 \mathrm{kgf} / \mathrm{cm}^{2}$. Las mediciones se realizaron en cada calicata a intervalos de $5 \mathrm{~cm}$ hasta los $30 \mathrm{~cm}$ de profundidad, ya que la mayor proporción de raíces de la pastura se encuentra a este nivel. La textura del suelo (fracciones de arena, limo y arcilla) se determinó por método del hidrómetro de Bouyoucos (Gee y Or, 2002) en el Laboratorio de Análisis de Suelos, Plantas, Agua y Fertilizantes de la Universidad Nacional Agraria la Molina (Lima).

\section{Humedad del suelo}

La humedad del suelo se midió por el método gravimétrico (Martínez, 1971). Para ello, se tomó una alícuota de 10,20 y $30 \mathrm{~cm}$ de profundidad en cada calicata. Las muestras se remitieron al laboratorio indicado anteriormente, donde fueron colocados en estufa a $105^{\circ} \mathrm{C} / 24 \mathrm{~h}$. El porcentaje de humedad gravimétrica se estimó de la siguiente manera: Humedad $(\%)=[($ Psh - Pss $) /$ Pss]*100, donde Psh: Peso de suelo húmedo (g); Pss: Peso de suelo seco (g).

\section{Temperatura y análisis químico del suelo}

- La temperatura del suelo se midió con un geotermómetro (General ${ }^{\circledR}$ T300-36, de 0.1 ${ }^{\circ} \mathrm{C}$ de precisión) bajo la copa de los árboles y fuera de ella, a una profundidad de 15 $\mathrm{cm}$ desde la superficie del suelo, entre las 09:00 y 11:00 horas.

- Para el análisis químico del suelo, se colectó una alícuota (1 kg aproximadamente) de cada calicata bajo y fuera de la copa 
de los árboles, entre 5 y $30 \mathrm{~cm}$ de profundidad. Las muestras fueron enviadas al Laboratorio de Análisis de Suelos, Plantas, Agua y Fertilizantes de la Universidad Nacional Agraria La Molina para su análisis.

- El pH del suelo se determinó usando un medidor de $\mathrm{pH}$ en suspensión de suelo/líquido de 1: 2.5 (Hendershot et al., 1993).

- La disponibilidad de fósforo se determinó utilizando el método de Olsen modificado (Olsen y Somers, 1982).

- El contenido de materia orgánica (MO) del suelo fue determinado en porcentaje por él método de Walkley y Black. Se basa en la oxidación de una muestra para determinar el contenido de carbono orgánico (CO). Se utilizó la siguiente ecuación: $\mathrm{MO}(\%)=$ $\% \mathrm{CO} \times 1.724$.

- La disponibilidad de potasio se determinó en partes por millón (ppm) por el método de acetato de amonio a pH 7 (Thomas, 1982).

- El fósforo se determinó en partes por millón (ppm) por el método de Olsen modificado. Extracto $\mathrm{NaHCO}_{3}$ a una concentración de $0.5 \mathrm{M}$ y pH 8.5.

- El aluminio se determinó en $\mathrm{cmolkg}^{-1}$, por el método de Yuan. Extracción con $\mathrm{KCl}$, N (USDA-NRCS, 1999).

\section{Precipitación}

Los valores de precipitación pluvial (mm) fueron recabados de la estación meteorológica de Jaén del Servicio Nacional de Meteorología e Hidrología (SENAMHI) para el año de evaluación (2018) (Figura 2).

\section{Análisis Estadístico}

Los resultados se analizaron mediante el diseño completamente al azar con arreglo factorial $3 \times 2$ utilizando el software estadístico R 3.6.1 con las librerías lsmeans y agricolae. Los factores fueron 3 ubicaciones del suelo (FCA, BCAj, BCAm) y 2 épo- cas del año (seca y lluviosa). Para la comparación de promedios se utilizó la prueba Duncan con un nivel de 0.05 para detectar diferencias estadísticas entre promedios.

\section{Resultados y Discusión}

\section{Clima y Características Físicas del Suelo}

La mayor precipitación fue en marzo (196.7 mm) y la menor en noviembre (15.6 $\mathrm{mm}$ ) (Figura 2). La producción de pastos fue afectada por los niveles de precipitación. Se reconoce que el crecimiento del pasto depende principalmente de la temperatura y humedad del suelo y régimen de lluvias a lo largo del año (López et al., 2018; Nuñez et al., 2019).

Se encontraron diferencias significativas $(p<0.05)$ entre la humedad del suelo en la época seca (10.99\%) y lluviosa (17.06\%), estando directamente relacionado al régimen de precipitación. La temperatura ambiental presentó diferencias significativas $(\mathrm{p}<0.05)$, registrándose los mayores valores en la época seca $\left(30.05^{\circ} \mathrm{C}\right)$ y fuera de la copa de los árboles $\left(30.82{ }^{\circ} \mathrm{C}\right)$ (Cuadro 1). La temperatura del suelo presentó diferencias significativas $(\mathrm{p}<0.05)$ entre sus tratamientos con valores de $31.20,28.90$ y $27.96{ }^{\circ} \mathrm{C}$ fuera de la copa, bajo la copa del árbol joven y mediano respectivamente (Cuadro 1). La presencia de los árboles disminuyó hasta $3.5^{\circ} \mathrm{C}$ en la temperatura ambiental y $3.2^{\circ} \mathrm{C}$ en la temperatura del suelo. Sin embargo, la humedad del suelo solo presentó diferencias significativas $(\mathrm{p}<0.05)$ entre fuera de la copa del árbol y bajo la copa del árbol mediano, probablemente debido a que presenta mayor canopia (Cuadro 1), favoreciendo a las pasturas, ya que el árbol disminuye su evapotranspiración (Ludwig et al., 2001). Este sistema genera un microclima que favorece al confort del animal. 
Cuadro 1. Variables climáticas y fiscas del suelo (media, \pm desviación estándar) en áreas de pastoreo de ganado con pasto castilla (Panicum maximum Jacq) y árboles faique (Acacia macracantha), en un fundo del distrito de Bellavista (Cajamarca, Perú, 2018)

\begin{tabular}{|c|c|c|c|c|c|c|}
\hline \multirow{2}{*}{\multicolumn{2}{|c|}{ Variables }} & \multicolumn{2}{|c|}{ Temperatura $\left({ }^{\circ} \mathrm{C}\right)$} & \multirow{2}{*}{$\begin{array}{c}\text { Humedad } \\
(\%)\end{array}$} & \multirow{2}{*}{$\begin{array}{c}\mathrm{DA} \\
\left(\mathrm{g} / \mathrm{cm}^{3}\right)\end{array}$} & \multirow{2}{*}{$\begin{array}{c}\text { Porosidad } \\
(\%)\end{array}$} \\
\hline & & Ambiente & Suelo & & & \\
\hline \multirow[t]{3}{*}{$\mathrm{U}$} & FCA & $30.82 \pm 5.2^{\mathrm{a}}$ & $31.20 \pm 4.7^{\mathrm{a}}$ & $12.98 \pm 3.3^{\mathrm{b}}$ & $1.40 \pm 0.11^{\mathrm{a}}$ & $46.91 \pm 4.3^{\mathrm{a}}$ \\
\hline & $\mathrm{BCAj}$ & $28.17 \pm 4.8^{b}$ & $28.90 \pm 3.0^{\mathrm{b}}$ & $13.87 \pm 4.5^{\mathrm{ab}}$ & $1.39 \pm 0.12^{\mathrm{a}}$ & $47.36 \pm 4.4^{\mathrm{a}}$ \\
\hline & BCAm & $27.23 \pm 4.8^{c}$ & $27.96 \pm 2.9^{c}$ & $15.23 \pm 4.6^{\mathrm{a}}$ & $1.41 \pm 0.14^{\mathrm{a}}$ & $46.75 \pm 5.1^{\mathrm{a}}$ \\
\hline \multirow[t]{2}{*}{$\mathrm{E}$} & Lluvia & $27.43 \pm 2.5^{b}$ & $26.63 \pm 3.0^{\mathrm{b}}$ & $17.06 \pm 2.9^{\mathrm{a}}$ & $1.34 \pm 0.11^{\mathrm{b}}$ & $49.34 \pm 4.4^{\mathrm{a}}$ \\
\hline & Seca & $30.05 \pm 5.4^{\mathrm{a}}$ & $32.08 \pm 4.2^{\mathrm{a}}$ & $10.99 \pm 1.9^{b}$ & $1.49 \pm 0.07^{\mathrm{a}}$ & $43.89 \pm 2.6^{\mathrm{b}}$ \\
\hline
\end{tabular}

U: ubicación, E: época de estudio, DA: densidad aparente, FCA: fuera de la copa de los árboles, BCAj: bajo la copa del árbol joven, BCAm: bajo la copa del árbol mediano $a, b, c$ Letras diferentes en cada columna revelan diferencias $(p<0.05)$ entre variables

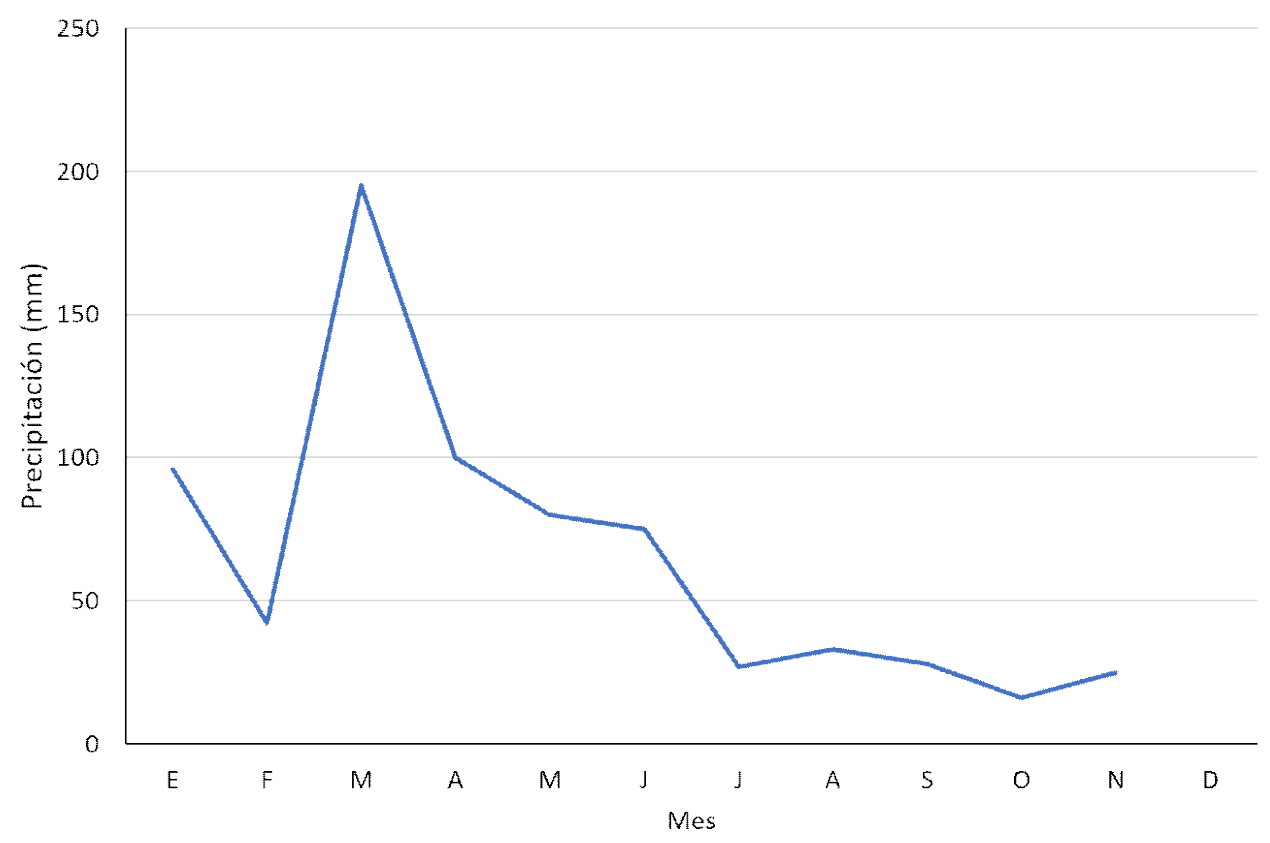

Figura 2. Curva de precipitación pluvial en la zona del estudio en 2018 


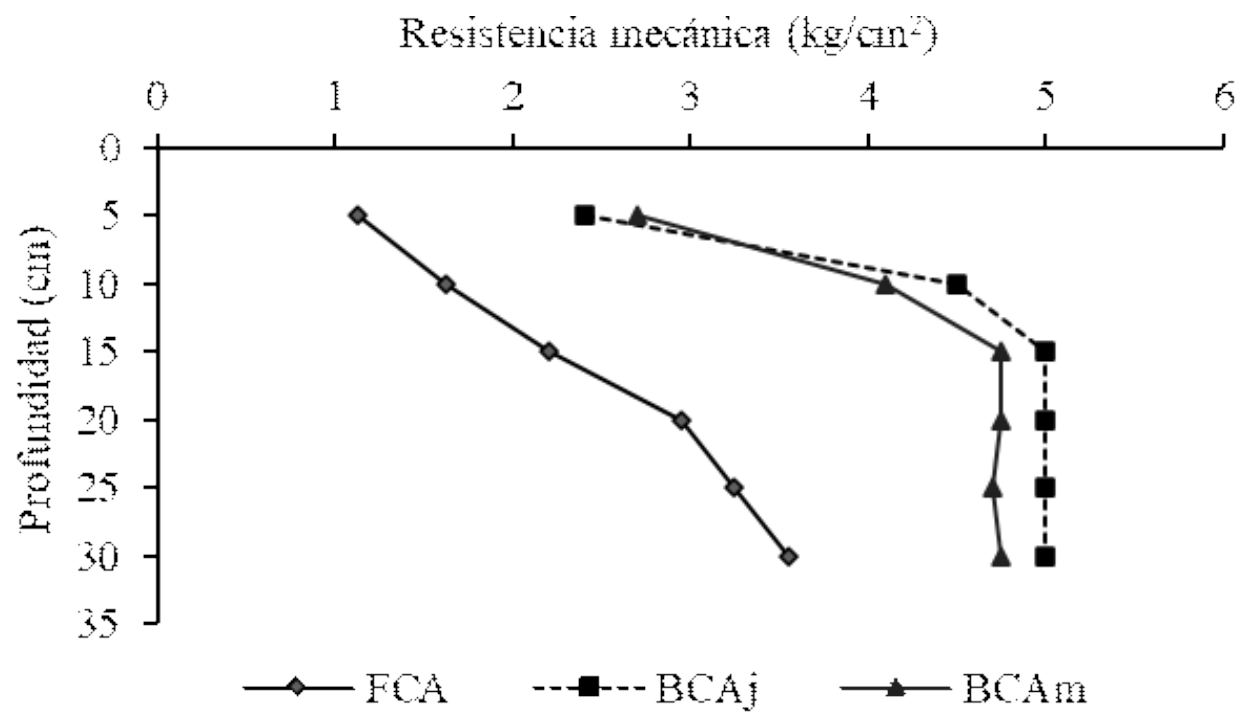

Figura 3. Variación de la resistencia mecánica en el suelo en época lluviosa en áreas de pastoreo de ganado con pasto castilla (Panicum maximum Jacq) y árboles faique (Acacia macracantha), en un fundo del distrito de Bellavista (Cajamarca, Perú, 2018). FCA: fuera de la copa de los árboles, BCAj: bajo la copa del árbol joven, BCAm: bajo la copa del árbol mediano

La densidad aparente del suelo no presentó diferencias significativas $(\mathrm{p}>0.05)$ por la ubicación; no obstante, se encontraron diferencias significativas $(\mathrm{p}<0.05)$ con valores de $1.34 \mathrm{~g} / \mathrm{cm}^{3}$ en lluviosa y $1.49 \mathrm{~g} / \mathrm{cm}^{3}$ en seca (Cuadro 1). La densidad aparente del suelo ligeramente mayor bajo la copa de los árboles medianos, se asocian a la mayor frecuencia de animales para tomar sombra, originando el pisoteo (Anderson et al., 2010); sin embargo, la materia orgánica que existe en suelo bajo la copa de los árboles promueve el ciclaje de nutrientes haciéndolo menos susceptible a la compactación (Siavosh et al., 2000).

La porosidad no presentó diferencia estadística en las tres ubicaciones del suelo; sin embargo, presentó diferencia significativa en las épocas del año con valores de 49.34 y $43.98 \%$ en la época lluviosa y seca, res- pectivamente (Cuadro 1). En sistemas de producción similar, se reportó una densidad aparente $\left(1.45 \mathrm{~g} / \mathrm{cm}^{3}\right)$ con árboles de pijuayo (Bactris gasipaes H.B.K) y pasto centrosema (Centrosema macrocarpum Benth) (Alegre et al., 2012).

La resistencia mecánica entre el árbol joven y mediano osciló entre 2.4 a 5.0 y 2.7 a $4.8 \mathrm{~kg} / \mathrm{cm}^{2}$, respectivamente, no presentando diferencias significativas entre sus promedios; sin embargo, tuvo mayor resistencia en el suelo bajo la copa del árbol joven. Fuera de la copa de los árboles los valores de resistencia mecánica oscilaron entre 1.17 y 3.52 $\mathrm{kg} / \mathrm{cm}^{2}$ (Figura 3). Similares resultados fueron reportados por Siavosh et al. (2000) al estudiar el suelo de una ganadería extensiva productora de leche en los Andes colombianos, cuyo valor de resistencia mecánica fue de $2.62 \mathrm{~kg} / \mathrm{cm}^{2}$. 


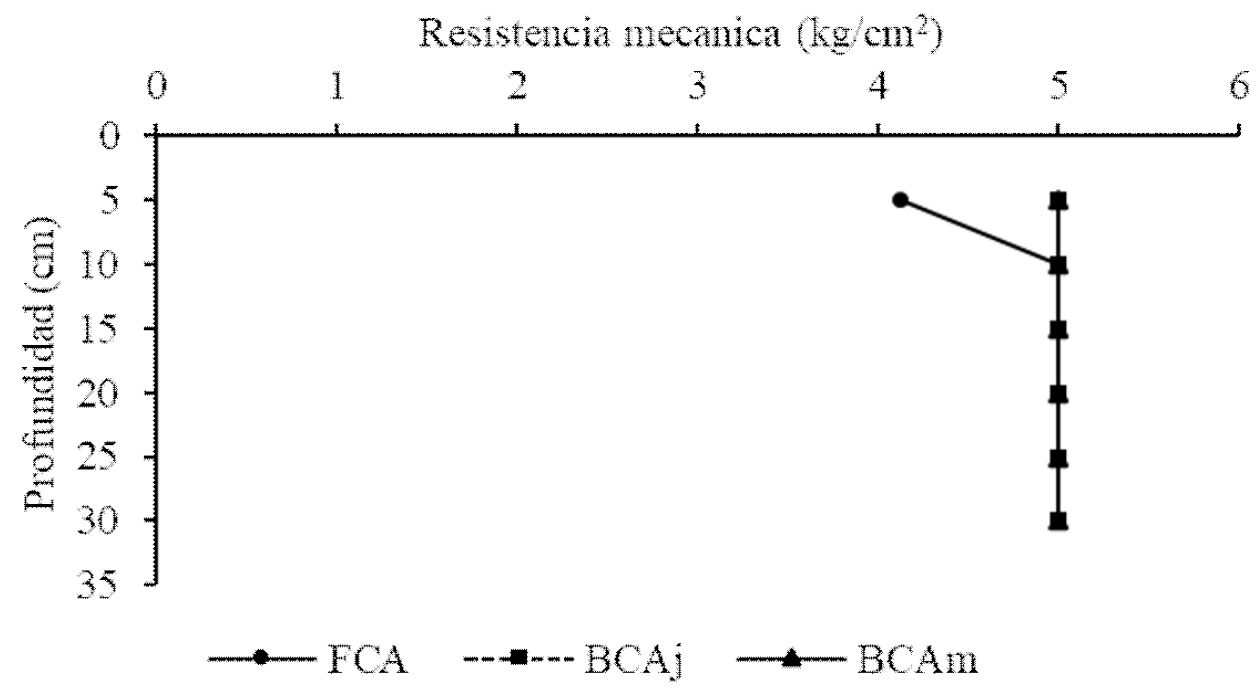

Figura 4. Variación de la resistencia mecánica en el suelo en época seca en áreas de pastoreo de ganado con pasto castilla (Panicum maximum Jacq) y árboles faique (Acacia macracantha), en un fundo del distrito de Bellavista (Cajamarca, Perú, 2018). FCA: fuera de la copa de los árboles, BCAj: bajo la copa del árbol joven, BCAm: bajo la copa del árbol mediano

En la Figura 4, la resistencia mecánica del suelo bajo la copa de los árboles y fuera de la copa de los árboles en la época seca no mostró diferencias $(\mathrm{p}>0.05)$ en todo el perfil del suelo con excepción de los primeros $5 \mathrm{~cm}$ de profundidad. La menor resistencia en la parte superficial del suelo puede deberse a la presencia de gramíneas y hojarasca producto de la caducifolicidad de ambos componentes del sistema (pasto y árbol). Evaluaciones realizadas por Lok et al. (2006) en suelo de sistemas silvopastoriles reportan comportamientos similares.

\section{Características Químicas y Fertilidad del Suelo}

El pH del suelo presentó diferencias significativas $(p<0.05)$, siendo menor bajo la copa del árbol mediano (7.83), posiblemente debido a la alta tasa de reciclaje de nutrientes por el comportamiento caducifolio de la $A$. macracantha. Comportamiento similar re- portaron Ferrari y Wall (2015) al evaluar el efecto del Prosopis juliflora de 6 años donde el pH del suelo disminuyó de 10.2 a 7.2. Sin embargo, Mendoza et al. (2000) comparando cultivos de Panicum maximum con árboles de Cordia elaeagnoides en suelos entisoles reportan $\mathrm{pH}$ de 6.81 a 7.45, respectivamente, indicando que los árboles tratan de neutralizar el pH. En el presente estudio, los árboles tuvieron la tendencia de neutralizar el pH del suelo llevando de 8.21 a 7.83 fuera de la copa del árbol y bajo la copa del árbol mediano, respectivamente (Cuadro 2). De otra parte, Mejía et al. (2018) y Bugarín et al. (2010) mencionan que los sistemas silvopastoriles corrijen los niveles de $\mathrm{pH}$ en el suelo.

La materia orgánica (\%) en el suelo no presentó diferencias significativas entre los grupos en estudio, aunque se pudo notar un ligero aumento bajo la copa de los árboles, debido a que el faique es una especie caduci- 
Cuadro 2. Suelo cubierto por pasto Panicum maximum Jacq. bajo las copas de árboles Acacia macracantha en áreas de pastoreo de ganado en un fundo del distrito de Bellavista (Cajamarca, Perú, 2018).

\begin{tabular}{lcccccc}
\hline \multirow{2}{*}{ Indicador } & \multicolumn{2}{c}{ FCA } & \multicolumn{2}{c}{ BCAj } & \multicolumn{2}{c}{ BCAm } \\
\cline { 2 - 7 } & Media & EE & Media & EE & Media & EE \\
\hline $\mathrm{pH}$ & $8.21^{\mathrm{a}}$ & 0.46 & $8.14^{\mathrm{b}}$ & 0.49 & $7.83^{\mathrm{c}}$ & 0.37 \\
Textura & FrArA & - & FrArA & - & FrArA & - \\
$\mathrm{MO}(\%)$ & $3.15^{\mathrm{a}}$ & 1.35 & $3.96^{\mathrm{a}}$ & 2.09 & $4.06^{\mathrm{a}}$ & 2.02 \\
$\mathrm{P}_{2} \mathrm{O}_{5}\left(\mathrm{~kg} \mathrm{ha}^{-1}\right)$ & $55.1^{\mathrm{b}}$ & 17.40 & $41.3^{\mathrm{b}}$ & 3.30 & $79.2^{\mathrm{a}}$ & 8.40 \\
$\mathrm{~K}_{2} \mathrm{O}\left(\mathrm{kg} \mathrm{ha}^{-1}\right)$ & $1126.4^{\mathrm{b}}$ & 263.70 & $980^{\mathrm{b}}$ & 286.40 & $2033.1^{\mathrm{a}}$ & 443.30 \\
$\mathrm{Al}^{+3} \mathrm{H}^{+}$ & 0 & - & 0 & - & 0 & - \\
\hline
\end{tabular}

FCA: fuera de la copa de los árboles, BCAj: bajo la copa del árbol joven, BCAm: bajo la copa del árbol mediano. EE: Error estándar, $\operatorname{Fr}$ Ar A: franco-arcillo-arenoso $a, b, c$ Letras diferentes en cada fila indican diferencias significativas $(p<0.05)$

folia, reciclando de esta forma la materia orgánica a través de la descomposición de la hojarasca; comportamiento similar reportado en otros estudios (Beukes y Cowling, 2003; Shi et al., 2010).

El contenido de $\mathrm{P}_{2} \mathrm{O}_{5}(\mathrm{~kg} / \mathrm{ha})$ y de $\mathrm{K}_{2} \mathrm{O}$ $(\mathrm{kg} / \mathrm{ha})$ fue significativamente mayor $(\mathrm{p}<0.05)$ en el suelo bajo la copa del árbol mediano en comparación con el contenido en el suelo bajo la copa del árbol joven y fuera de la copa de los árboles (Cuadro 2). Por su parte, Villagra et al. (2004) encontraron que la presencia de Prosopis flexulosa mejora las condiciones de materia orgánica, nitrógeno y fosforo bajo la copa del árbol, mientras no afecta el contenido de potasio y el valor del $\mathrm{pH}$. En este sentido, Andrade et al. (2008), reportaron que Acacia mangium deposita la mayor cantidad de material vegetal en forma de hojarasca, al ser comparado con otro grupo de plantas arbóreas, mientras que Obispo et al. (2008) reportaron que la fertilidad, porosidad y capacidad de almacenamiento de agua en el suelo se mejora bajo los árboles.
No se detectaron niveles de aluminio en los suelos debido que son suelos ligeramente alcalinos con formación geológica basada en roca basáltica básica, siendo su clasificación taxonómica vertisol con predominancia de arcilla montmorillonita (Ditzler, 2017). Similares resultados fueron reportados por Nuñez (2019) en la misma zona geográfica. Este elemento se encuentra generalmente en las arcillas y bajo ciertas condiciones se puede volver tóxico para las plantas, ya que inhibe el desarrollo de las raíces. Esto ocurre generalmente en suelos con pH menor a 5 (Zapata, 2004). La presencia de aluminio implica una reducción de la fertilidad debido a que producen fenómenos de degradación de la materia orgánica y pérdida de nutrientes (Acevedo, 2007).

\section{Conclusiones}

- La Acacia macracantha mejora la humedad, materia orgánica, niveles fósforo y potasio en el suelo; sin embargo, 
debido a la acumulación de materia orgánica, el pH disminuyó ligeramente.

- La mayor resistencia mecánica bajo la copa de los árboles encontrada se debe al comportamiento animal, ya que este busca sombra durante las horas de mayor temperatura ambiental. Sin embargo, fuera de la copa del árbol esta característica física es muy variable, especialmente en la época seca.

\section{Literatura Citada}

1. Acevedo O. 2007. Aluminio, un indicador de calidad ambiental en suelos de carga variable. Doctoral. México: Univ. Autónoma del Estado de Hidalgo. 145 p.

2. Alegre JC, Vega R, La Torre B. 2012. Manual de manejo de suelos con sistemas silvopastoriles. Lima, Perú: Univ. Nacional Agraria La Molina. 28 p.

3. Anderson PML, Hoffman MT, O'Farrell P. 2010. Above ground perennial plant biomass across an altitudinal and land-use gradient in Namaqualand, South Africa. S Afr J Botany 76: 471-481. doi: 10.1016/ j.sajb.2010.03.004

4. Andrade HJ, Brook R, Ibrahim M. 2008. Growth, production and carbon sequestration of silvopastoral systems with native timber species in the dry lowlands of Costa Rica. Plant Soil 308: 11-22.

5. Beukes PC, Cowling RM. 2003. Nonselective grazing impacts on soilproperties of the Nama Karoo. Afr J Range Forage Sci 56: 547-552. doi: 10.2989/10220110009485736

6. Bugarín J, Bojórquez JI, Lemus C, Murray RM, Ontiveros H, Aguirre J, Hernández A. 2010. Comportamiento de algunas propiedades físicoquímicas del suelo con diferente sistema silvopastoril en la llanura norte de Nayarit. Cultrop 31(2).
7. Dagang AB, Nair PKR. 2003. Silvopastoral research and adoption in Central America: recent findings and recommendations for future directions. Agroforestry Systems 59: 149-155.

8. Ditzler CA. 2017. Propiedades y clasificación del suelo (taxonomía del suelo). En: The soils of the USA. USA: Springer. p. 29-41.

9. [FAO] Organización de las Naciones Unidas para la Agricultura y la Alimentación. 1992. Forests, trees and food [Internet]. Available in: http:// www.fao.org/3/u5620e/U5620E05.htm\#TopOfPage

10. [FAO] Organización de la Naciones Unidas para la Agricultura y la Alimentación. 2004. Inventario forestal nacional. Manual de campo modelo. Guatemala. [Internet]. Disponible en: http:// www.fao.org/3/a-ae578s.pdf

11. Ferrari AE, Wall LG. 2015. Utilización de árboles fijadores de nitrógeno para la revegetación de suelos degradados. Rev Fac Agron La Plata 105: 63-87.

12. Gee GW, Or D. 2002. Particle size analysis. In: Dan DJ, Topps GC (eds). Methods of soil analysis. Part 4. Physical methods. Madison, USA: Soil Sci. Soc. of America Book Series. p 225-293.

13. Grossman RB, Reinch TG 2002. Bulk density and linear extensibility. In: Dan DJ, Topps GC (eds). Methods of soil analysis. Part 4. Physical methods. Madison, USA: Soil Sci. Soc. of America Book Series. p 201-228.

14. Hendershot WH, Lalande $\mathrm{H}$, Duquette M. 1993. Soil reaction and exchangeable acidity. In: Carter, M.R (Ed.). Soil Sampling and Methods of Soil Analysis. Canadian Society of Soil Science, Lewis Publishers, London. 141 - 145.

15. Lemaire G, Franzluebbers $A$, de Faccio Carvalho PC, Dedieu B. 2014. Integrated crop-livestock systems: strategies to achieve synergy between agricultural production and environmental quality. Agric Ecosyst Environ 190: 4-8. doi: 10.1016/j.agee.2013.08.009. 
16. Lok $S$, Crespo G, Frómeta E, Fraga $S .2006$. Estudio de indicadores de estabilidad del pasto y el suelo en un sistema silvopastoril con novillas lecheras. Rev. Cubana Cienc Agríc 40: 229-237.

17. López AG, Nuñez DJ, Aguirre TL, Flores $M E$. 2018. Dinámica de la producción primaria y valor nutritivo de tres gramíneas tropicales (Melinis minutiflora, Setaria sphacelata y Brachiaria mutica) en tres estados fenológicos. Rev Inv Vet Perú 29: 396-409. doi: 10.15381/ rivep.v29i2.14494

18. Ludwig F, De Kroon H, Prins $H$, Berendse F. 2001. Effects of nutrients and shade on tree-grass interaction in an East African savanna. J Veg Sci 12: 579588. doi: 10.2307/3237009

19. Martínez M. 1971. Calibración y comparación de algunos métodos para la determinación de humedad del suelo. Tesis de Maestría. Chapingo, México: Colegio de Postgraduados. $120 \mathrm{p}$.

20. Mejía MJ, Congo CD, Velástegui FJ, Vera A. 2018. Influencia de los sistemas silvopastoriles en el mejoramiento del $\mathrm{pH}$ del suelo. I Congreso Internacional Alternativas Tecnológicas para la Producción Agropecuaria Sostenible en la Amazonía Ecuatoriana. La Joya de los Sachas, Ecuador.

21. Mendoza MN, Galicia L, Oliva FG. 2000. Efecto de dos especies de árboles remanentes y de un pasto en la capacidad amortiguadora del $\mathrm{pH}$ del suelo en un ecosistema tropical estacional. Botanical Sci 67: 17-24. doi: 10.17129/ botsci.1624

22. Moraes A, Carvalho F, Anghinoni I, Lustosa SBC, De Costa A, Kunrath TR. 2014. Integrated crop-livestock systemas in the Brazilian subropics. Eur J Agron 57: 4-9. doi: 10.1016/j.eja.2013.10.004

23. Nuñez J. 2017. Perfil alimentario y plan de pastoreo para la producción lechera con pasturas Panicum maximum Jacq. Tesis de Maestría. Lima, Perú: Univ. Nacional Agraria La Molina. 125 p.
24. Nuñez Delgado J, Ñaupari Vásquez J, Flores Mariazza E. 2019. Comportamiento nutricional y perfil alimentario de la producción lechera en pastos cultivados (Panicum maximum Jacq). Rev Inv Vet Perú 30: 178-192. doi: 10.15381/ rivep.v30i1.15681

25. Obispo N, Espinoza Y, Gil J, Ovalles F, Rodriguez M. 2008. Efecto del sombreado sobre la producción y calidad del pasto guinea (Panicum maximum) en un sistema silvopastoril. Zootec Trop 26: 285-288.

26. Oliva S. 2016. Influencia de factores socioeconómicos y ambientales sobre la adopción de tecnologías silvopastoriles por productores ganaderos, distrito de Molinopampa, Amazonas. Tesis de Maestría. Lima, Perú: Univ. Nacional Agraria La Molina. $102 \mathrm{p}$.

27. Olsen SR, Sommer LE, 1982. Phosphorus. In: Page AL, Miller RH, Keeney DR. (eds). Methods of soil analysis. Part 4. Physical methods. Madison, USA: Soil Sci. Soc. of America Book Series. p 403-430.

28. [SENAMHI] Servicio Nacional de Meteorología e Hidrología. 2018. Lima: Ministerio del Ambiente. [Internet]. Disponible en: https://www.senamhi.gob.pe/?\&p=descarga-datoshidrometeorologicos

29. Shi F, Chen H, Wи Y, Wи N. 2010. Effects of livestock exclusion on vegetation and soil properties under two topographic habitats in an alpine meadow on the eastern Qinghat-Tibetan Plateau. Polish J Ecol 58: 125-133.

30. Siavosh S, Rivera J, Gómez M. 2000. Impacto de sistemas de ganadería sobre las características físicas, químicas y biológicas de suelos en los Andes de Colombia. En: Conferencia de la FAO sobre Agroforestería para la Producción Animal en Latinoamérica. Cali, Colombia: FAO-CIPAV.

31. Thomas GW. 1982. Exchangeable cations. In: Page AL, Miller A, Keeney DR. $2^{\text {nd }}$ ed. Methods of soil analysis. Part 
2. Physical methods. Madison, USA: Soil Sci. Soc. of America Book Series. p 159166.

32. USDA-NRCS. 1999. Taxonomía de suelos: un sistema básico de clasificación de suelos para realizar e interpretar levantamientos de suelos. Manual de agricultura. USA: USDA. $436 \mathrm{p}$.

33. Villagra PE, Cony MA, Mantován NG, Rossi BE, González Loyarte MM, Villalba R, Marone L. 2004. Ecología y manejo de los algarrobales de la Provincia Fitogeográfica del Monte. Mendoza, Argentina: IANIGLA CONICET. $32 \mathrm{p}$.

34. Yates C, Dorward P, Hemery G, Cook P. 2007. The economic viability and potential of a novel poultry agroforestry system. Agroforestry Systems 69: 13-28.

35. Zapata H. 2004. Química de la acidez del suelo. Medellín. Colombia: Univ. Nacional de Colombia. p 137. 